\title{
ON WRONSKIANS WHOSE ELEMENTS ARE ORTHOGONAL POLYNOMIALS ${ }^{1}$
}

\section{ROBERT VERMES}

In a recent paper S. Karlin and G. Szegö [1], among many other topics, proved theorems concerning the sign and real zeros of the Wronskian

$$
W(n, l ; x)=\left|\begin{array}{cccc}
Q_{n}(x) & Q_{n+1}(x) & \cdots & Q_{n+l-1}(x) \\
Q_{n}^{\prime}(x) & Q_{n+1}^{\prime}(x) & \cdots & Q_{n+l-1}^{\prime}(x) \\
\vdots & \vdots & \vdots \\
Q_{n}^{(l-1)}(x) & Q_{n+1}^{(l-1)}(x) & \cdots & Q_{n+l-1}^{(l-1)}(x)
\end{array}\right|
$$

where $\left\{Q_{r}(x)\right\}(r=0,1,2, \cdots)$ is a sequence of orthogonal polynomials associated with a certain measure. They proved $[1$, Theorem $1]$ if $l$ is even, $W(n, l ; x)$ keeps constant sign for all real $x$, i.e., the Wronskian (1), which is an $n . l$ degree polynomial, has only complex roots. In this note we want to show that if $\left\{Q_{r}(x)\right\}$ are orthogonal polynomials on a finite interval, we can obtain additional information on the location of all the zeros of (1).

TheOREM 1. Let $q(x)$ be a positive continuous function on the finite interval $a \leqq x \leqq b$; and let $\left\{Q_{r}(x)\right\}(r=0,1,2, \cdots)$ be a sequence of polynomials ${ }^{2}$ which satisfy $\int_{a}^{b} q(x) Q_{n}(x) Q_{m}(x) d x=0, n \neq m$, then all the zeros of the Wronskian (1) are in $S=S[(a, b) ; \pi / l]$, where $S[(a, b) ; \Phi]$ denotes the region comprised of all points at which the interval $[a, b]$ subtends an angle of at least $\Phi$.

For the proof of Theorem 1 we need the following:

LEMMA I. Let $\rho(x)$ be an $m$ th degree polynomial; let $\alpha(x) \geqq 0, a \leqq x \leqq b$ be continuous and suppose

$$
\int_{a}^{b} \alpha(x) \rho(x) d x=0 .
$$

Then $\rho(x)$ has at least one zero in $S[(a, b), \pi / m]$.

The above lemma is a special case of Marden's Mean-Value theo-

Received by the editors June 15, 1962 and, in revised form, September 27, 1962.

1 This research was supported, in part, by NSF Grant G-16315.

${ }^{2} Q_{r}(x)$ denotes a polynomial of the exact degree $r$. 
WRONSKIANS WHOSE ELEMENTS ARE ORTHOGONAL POLYNOMIALS 125

rem $[2$, p. 87 (Theorem 24.3)]. The proof for $\alpha(x) \equiv 1$ can be found in $[3]$.

With the aid of Lemma I we prove

Lemma II. Let $\left\{Q_{r}(x)\right\}(r=0,1,2, \cdots)$ be as in Theorem 1 , then for any complex numbers $a_{v}$ the polynomial:

$$
P(x)=\sum_{\nu=0}^{l-1} a_{\nu} Q_{n+\nu}(x)
$$

has at least $n$ zeros in $S[(a, b) ; \pi / l]$.

Proof. Suppose that there are only $k \leqq n-1$ zeros $\zeta_{1}, \zeta_{2}, \cdots, \zeta_{k}$ of $P(x)$ in $S$, and the remaining zeros $\eta_{k+1}, \eta_{k+2}, \cdots, \eta_{n+l-1}$ are outside. Make use of Lemma I, letting

(4) $\alpha(x)=q(x)\left[\left(x-\zeta_{1}\right)\left(x-\bar{\zeta}_{1}\right)\right] \cdots\left[\left(x-\zeta_{k}\right)\left(x-\bar{\zeta}_{k}\right)\right]$

$$
\cdot\left[\left(x-\eta_{k+1}\right)\left(x-\bar{\eta}_{k+1}\right)\right] \cdots\left[\left(x-\eta_{n-1}\right)\left(x-\bar{\eta}_{n-1}\right)\right],
$$

(5) $\rho(x)=\left(x-\eta_{n}\right)\left(x-\eta_{n+1}\right) \cdots\left(x-\eta_{n+l-1}\right)$.

From the definition of $\alpha(x)$ in (4) it is clear that $\alpha(x) \geqq 0$ for $a \leqq x \leqq b$. The functions in (4), (5) also satisfy (2), for

$$
\rho(x) \alpha(x)=q(x)\left[\left(x-\zeta_{1}\right) \cdots\left(x-\zeta_{k}\right)\left(x-\eta_{k+1}\right) \cdots\left(x-\eta_{n+l-1}\right)\right]
$$

$$
\cdot\left[\left(x-\bar{\zeta}_{1}\right) \cdots\left(x-\bar{\eta}_{n-1}\right)\right]
$$

$$
=c\left[q(x) \sum_{\nu=0}^{l-1} a_{\nu} Q_{n+\nu}(x)\right] \pi_{n-1}(x) \quad(c \neq 0)
$$

where

(7) $\pi_{n-1}(x)=\left(x-\bar{\xi}_{1}\right)\left(x-\bar{\xi}_{2}\right) \cdots\left(x-\bar{\xi}_{k}\right)\left(x-\bar{\eta}_{k+1}\right) \cdots\left(x-\bar{\eta}_{n-1}\right)$

is an $n-1$ degree polynomial and we obtain:

$$
\int_{a}^{b} \alpha(x) \rho(x) d x=c \sum_{\nu=0}^{l-1} a_{\nu} \int_{a}^{b} q(x) Q_{n+\nu}(x) \pi_{n-1}(x) d x=0 .
$$

We conclude from Lemma I that $\rho(x)$ has at least one zero in $S[(a, b) ; \pi / l]$, which contradicts the definition of $\rho(x)$.

For the proof of Theorem 1 assume that there is at least one zero $z_{0}$ which is not in $S$, then the system of $l$ linear equations in $a_{\eta}$ :

$$
\sum_{\nu=0}^{l-1} a_{\nu} Q_{n+\nu}^{(m)}\left(z_{0}\right)=0 \quad(m=0,1,2, \cdots, l-1)
$$

has a nontrivial solution $\left(W\left(n, l ; z_{0}\right)=0\right)$. Consider the $n+l-1$ degree polynomial 


$$
P(x)=\sum_{\nu=0}^{l-1} a_{\nu} Q_{n+\nu}(x)
$$

which by (9) has an $l$-fold zero at $x=z_{0}$. By our lemma the polynomial (10) has at least $n$ zeros in $S$, i.e., the $n+l-1$ degree polynomial $P(x)$ has at least $n+l$ zeros, which is impossible. Theorem 1 is thus proved.

From Theorem 1 we obtain by elementary geometrical consideration the following

Corollary I. Let $\left\{Q_{r}(x)\right\}(r=0,1,2, \cdots)$ be as in Theorem 1 , then $W(n, l ; x)$ has no zeros in the sectors: $\pi-\pi / l<\operatorname{Arg}(z-a)<\pi+\pi / l$ and $-\pi / l<\operatorname{Arg}(z-b)<\pi / l$.

\section{REMARKS.}

(1) Theorem 1 gives no information about the existence of real zeros in the interval $(a, b)$. In the above mentioned paper [ 1 , Theorem 2] of Karlin and Szegö it is proved that in case $l$ odd $W(n, l ; x)$ has exactly $n$ simple zeros in $(a, b)$.

(2) The region $S[(a, b) ; \pi / l]$ is independent of $n$. For example, if $l=2$, by using the Christoffel-Darboux formula, we obtain the following

CorollaRy II. If $\left\{Q_{r}(x)\right\}(r=0,1,2, \cdots)$ of Theorem 1, are also orthonormal, then all the zeros of

$$
K_{n}(x)=\sum_{\nu=0}^{n}\left[Q_{\nu}(x)\right]^{2}=c_{n} W(n, 2 ; x) \quad(n=1,2, \cdots)
$$

are in the circle $|x-(a+b) / 2| \leqq(b-a) / 2$.

\section{REFERENCES}

1. S. Karlin and G. Szegö, On certain determinants whose elements are orthogonal polynomials, J. Analyse Math. 8 (1960/61), 1-157.

2. M. Marden, The geometry of the zeros of a polynomial in a complex variable, Math. Surveys No. 3, Amer. Math. Soc., Providence, R. I., 1949.

3. M. Fekete, Ueber Gebiete, in denen Komplexe Polynome jeden Wert zwischen zwei gegebenen annehmen, Math. Z. 22 (1925), 1-7.

UNIVERSity OF WisCoNSIN-MilwaUkeE 